\title{
Prognostic value of immune scores in the microenvironment of colorectal cancer
}

\author{
WENLIANG YUAN ${ }^{1}$, WEI CAI ${ }^{2,3}$, XIAO HUANG $^{4}$ and SIHUA PENG ${ }^{2,3}$ \\ ${ }^{1}$ Department of Data Science, College of Mathematics and Information Engineering, Jiaxing University, Jiaxing, \\ Zhejiang 314001; ${ }^{2}$ Key Laboratory of Exploration and Utilization of Aquatic Genetic Resources (Shanghai \\ Ocean University), Ministry of Education; ${ }^{3}$ International Research Center for Marine Biosciences at Shanghai \\ Ocean University, Ministry of Science and Technology, Shanghai 200093; ${ }^{4}$ Department of Data Technology, \\ College of Mathematics and Computer Science, Chizhou University, Chizhou, Anhui 247000, P.R. China
}

Received February 28, 2020; Accepted August 18, 2020

DOI: $10.3892 / \mathrm{ol} .2020 .12119$

\begin{abstract}
Cancer immunotherapy has become an important means of cancer treatment; however, the complex composition and heterogeneity of the colorectal cancer (CRC) microenvironment pose a huge challenge to cancer immunotherapy. Using data downloaded from The Cancer Genome Atlas database, the differences in the microenvironment between cases with low and high immune scores were examined at the multiomics level using bioinformatics approaches. It was revealed that the samples with high immune scores had good cytolytic immune responses and relatively abundant stromal cells, as well as significant infiltration of 22 immune cell subsets and a high non-synonymous mutation burden and neoantigen burden. All of these characteristics contribute to a good prognosis. To better understand the impact of immune-related genes on prognosis, differentially expressed genes between the low and high immune score samples were identified and it was concluded that serpin family Emember 1 (SERPINE1) and ubiquitin C-terminal hydrolase L1 (UCHL1) may be potential therapeutic targets. The relationship between the immune score and the infiltration of 22 immune cells and the difference in SERPINE1 expression were verified by analyzing the GSE17536 and GSE21510 data sets downloaded from the Gene Expression Omnibus database.The present study analyzed the
\end{abstract}

Correspondence to: Dr Wenliang Yuan, Department of Data Science, College of Mathematics and Information Engineering, Jiaxing University, 56 Yuexiu South Road, Jiaxing, Zhejiang 314001, P.R. China

E-mail: liuli0355@zjxu.edu.cn

Professor Sihua Peng, Key Laboratory of Exploration and Utilization of Aquatic Genetic Resources (Shanghai Ocean University), Ministry of Education, 999 Hucheng Ring Road, Shanghai 200093 , P.R. China

E-mail: shpeng_haida@sina.com

Key words: colorectal cancer, cancer immunotherapy, tumor microenvironment, immune scores, neoantigen burden, prognosis unique properties of immune cells in the CRC microenvironment, which are of great significance for understanding CRC immune mechanism and may also provide novel ideas for the targeted design of cancer immunotherapy.

\section{Introduction}

Colorectal cancer (CRC) has the second-highest mortality rate among all cancers, and its associated mortality ranks fourth $(9.0 \%)$ and third $(9.5 \%)$ among all cancers in male and female patients with cancer, respectively (1). In clinical practice, surgical resection is a common treatment for CRC (2). However, approximately $50-60 \%$ of patients diagnosed with CRC have metastasis and $80-90 \%$ of them are unresectable liver metastases $(3,4)$. In recent years, cancer immunotherapy has become an important means of cancer treatment (5), and it is one of the hotspots in the field of cancer research. However, the complex components and heterogeneity of the tumor microenvironment pose a huge challenge to cancer immunotherapy.

The tumor microenvironment, which is composed of molecules such as immune cells and mesenchymal cells, is the cell environment in which the tumor is located (6). A pan-immune immunogenomic analysis revealed that numerous tumor-infiltrating lymphocytes associated with adaptive immunity are associated with a good prognosis, including activated $\mathrm{CD}^{+}$ $\mathrm{T}$ cells, resting memory $\mathrm{CD} 4^{+} \mathrm{T}$ cells and effector memory $\mathrm{CD}^{+} \mathrm{T}$ cells (7). To achieve precise immunotherapy, studies have defined the tumor mutation burden (TMB) by the number of mutations per megabase of DNA, as a predictive biomarker for evaluation (8). The higher the TMB is associated with the likelihood of the tumor being sensitive to immunotherapies (9). Immune cells are the major non-tumor component of a tumor microenvironment and have been indicated to be valuable for the diagnosis and prognostic assessment of patients with CRC (10). Recently, an algorithm called ESTIMATE, which calculates an immune score based on specific gene expression characteristics of immune cells, was used to predict the infiltration of non-tumor cells (11). Subsequent studies have demonstrated the effectiveness of this big data-based algorithm (12-14). 
Using the CRC cohort data from The Cancer Genome Atlas (TCGA) database and immune scores derived from the ESTIMATE algorithm, the present study investigated whether tumors with a higher mutation burden, neoantigen burden and a greater infiltration degree of 22 immune cell subsets are associated with higher immune scores.

\section{Materials and methods}

Data sources and preprocessing. All genomic, clinical and mutation annotation format (MAF) data were obtained from TCGA CRC cohorts in February 2019 according to the following specific parameters: The major site was the colon or rectum and the experimental strategy was RNA sequencing. First, the samples with survival times of $<30$ days were discarded and the samples without complete clinical information or MAF data were removed. Finally, the data of 432 tumor samples with 18 matched normal samples were retained.

The gene expression data in fragments per kilobase of transcript per million mapped reads format were also retrieved from TCGA and converted to transcripts per million (TPM). The immune scores and stromal scores were calculated by using the ESTIMATE algorithm based on the expression values in TPM (11). By comparing the differences in overall survival (OS), the optimal threshold for immune score grouping was determined. When the patient's immune score was above this threshold, the sample was assigned to the high immune score (HIM) group and otherwise to the low immune score (LIM) group.

The data used for verification were downloaded from Gene Expression Omnibus (GEO, https://www.ncbi.nlm.nih.gov/ geo/) and relevant datasets were identified using the following key words: ('colorectal cancer' OR 'colon cancer') AND ('prognosis' OR 'prognostic' OR 'survival') AND ('Homo sapiens'). Datasets obtained by sequencing using the GPL570 platform were selected and certain datasets were excluded. Duplicates and datasets with small sample sizes $(\mathrm{n}<50)$ were also excluded. The datasets GSE17536 and GSE21510 associated with CRC were finally selected and merged into a combined dataset. Gene probe names were transformed into gene names based on platform annotation files. Subsequently, the gene expression data for each sample and the corresponding clinical information were organized for further analysis.

Functional analysis in silico. The immune cytolytic activity scores were obtained by the geometric mean of the Granzyme A (GZMA) and Perforin 1 (PRF1) expression values in TPM (15). The data regarding microsatellite stable (MSS) or microsatellite instability-high (MSIH) status for 291 TCGA CRC samples were also obtained (16). The consensus molecular subtypes (CMSs) classification classifies CRC into four molecular subtypes (CMS1, CMS2, CMS3 and CMS4) with distinct biological characteristics. The samples in the HIM and LIM groups were classified according to the CMS system using the R package CMScaller (17).

The immune-associated genes were downloaded from the immunology database and analysis portal (ImmPort; https:// immport.niaid.nih.gov) (18). These immune-associated genes have a variety of roles in immune pathways. After filtering out the synonymous variants and variants in intergenic or noncoding regions, the maftools package (19) was used for mutation burden analyses and mutational spectral visualization. The neoantigens for each sample and neoantigen origin protein information were downloaded from The Cancer Immune Atlas (TCIA; https://tcia.at/home).

To evaluate tumor-infiltrating immune cell (TIIC) composition in CRC, the CIBERSORT deconvolution algorithm (20) was used to estimate the proportion of 22 immune cell types in HIM and LIM.

Differentially expressed mRNAs between samples from the HIM and LIM groups were screened using edgeR (21) with the criteria of $\log _{2}$ fold changel $>1.5$ and a false discovery rate (FDR) $<0.01$. The TIMER online database (22) was used to analyze and visualize the abundance of TIICs according to differentially expressed genes. Cytoscape software (23) (two plugins: ClueGO and CluePedia) was used for the Kyoto Encyclopedia of Genes and Genomes analyses and only pathways with $\mathrm{P}<0.05$ were considered. The GeneMANIA (24) plugin was also employed to investigate the functional association of the differentially expressed mRNAs between samples from the HIM and LIM groups.

Statistical analysis. All statistical analyses were performed using R software (version 3.5.0) and Bioconductor (https:// www.bioconductor.org/). An unpaired t-test was used to compare differences in various parameters (including stromal scores, cytolytic activity scores, and MSS vs. MSIH status) between samples from the HIM and LIM groups, and to compare the non-synonymous mutation burden in the HIM. A Wilcoxon rank sum test was used to examine differences in medians. Kaplan-Meier curve analyses using the survival package version 3.2 (25) were performed to analyze the association between the mRNA expression profiles and OS. $\mathrm{P}<0.05$ was considered to indicate statistical significance.

\section{Results}

Immune scores, sample grouping and demographic statistics. The gene expression profiles and clinical information of all 432 patients with CRC from the TCGA database were retrieved. Based on the ESTIMATE algorithm, the immune scores were distributed between -899.56 and 2,999.28 (Fig. 1A). The optimal threshold for dividing samples into the HIM and LIM groups was determined using the function surv_cutpoint of the survival package in $\mathrm{R}$ (cutoff=3.58). The detailed clinical and pathological characteristics of the study population in HIM and LIM, including age, sex, ethnicity, pathological stage, tumor (T) stage, nodal (N) stage and metastasis (M) stage, are summarized in Table I. The median age for all patients was 60 years (interquartile range, 31-90 years). Of the total patients, the HIM group contained $171(52.6 \%)$ male and $154(47.4 \%)$ female and the LIM group contained $24(22.4 \%)$ male and $83(77.6 \%)$ female.

Comparison of characteristics between the HIM and LIM groups. Immune scores represent the infiltration of immune cells in tumor tissues. It was indicated that MSIH tumors were associated with higher immune scores compared with MSS tumors $(\mathrm{P}<0.001$; Fig. 1B). Furthermore, samples in the HIM group had higher stromal scores than those in 

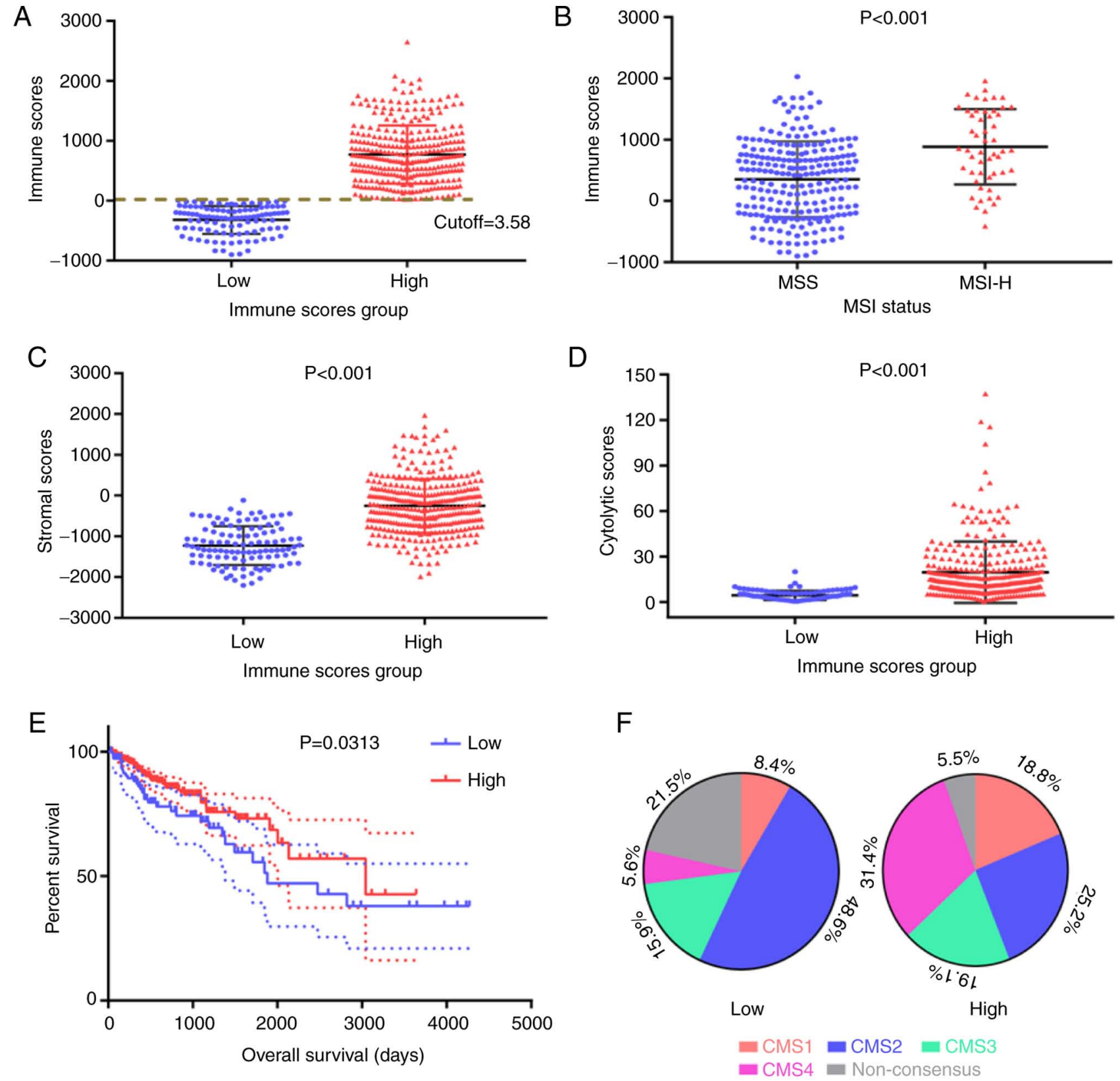

Figure 1. Characteristics of HIM and LIM samples. (A) Distribution of immune scores. CRC samples were divided into HIM and LIM samples based on their immune scores. (B) Immune scores in relation to MSS vs. MSIH status. (C) Distribution of stromal scores in HIM and LIM samples. (D) Distribution of cytolytic scores in the HIM and LIM samples. (E) Survival analysis of patients with CRC in the HIM and LIM groups. A vertical drop in the curves indicates an event. The dotted lines indicate the upper and lower limits of confidence. The area between the two broken lines represents the confidence interval. (F) Distribution of CMSs in HIM and LIM samples. CRC, colorectal cancer; HIM, high immune score; LIM, low immune score; MSS, microsatellite stability; MSIH, microsatellite instability-high; CMS, consensus molecular subtype.

the LIM group $(\mathrm{P}<0.001$; Fig. $1 \mathrm{C})$, indicating a positive association between immune and stromal scores. In addition, the association of the immune score and cytolytic activity score in CRC was examined. Of note, the distribution of cytolytic activity scores in the HIM and LIM groups was similar to that of the stromal scores, with a significantly higher score in the HIM group $(\mathrm{P}<0.001$; Fig. 1D).

A Kaplan-Meier survival curve analysis was also performed, indicating that OS in the HIM group was longer than that in the LIM group ( $\mathrm{P}=0.0313$ according to the log-rank test; Fig. 1E). CMSs based on gene expression profiles provide a biological stratification framework with great potential for biomarker development. The distribution of CMS subtypes in the HIM and LIM groups is presented in Fig. 1F. In the LIM group, CMS2 accounted for almost half of the cases (48.6\%), while in the HIM group, the other CMSs were comparatively more prevalent, particularly CMS4 (Fig. 1F).

Landscape of immune infiltration in HIM CRC. To further analyze the differences in immune cell invasion between the HIM and LIM groups, the CIBERSORT algorithm was used to investigate the differences in the proportion of 22 immune cell subsets between samples from the two groups. The differences in the proportion of 22 immune cell types detected in all 
Table I. Clinicopathological features of the patients with colorectal cancer $(n=432)$.

\begin{tabular}{|c|c|c|}
\hline Subtype & $\begin{array}{c}\text { HIM } \\
(n=325)\end{array}$ & $\begin{array}{c}\text { LIM } \\
(\mathrm{n}=107)\end{array}$ \\
\hline \multicolumn{3}{|l|}{ Age (years) } \\
\hline$>60$ & $243(74.8)$ & $74(69.2)$ \\
\hline$\geq 60$ & $82(25.2)$ & $33(30.8)$ \\
\hline \multicolumn{3}{|l|}{ Sex } \\
\hline Male & $171(52.6)$ & $24(22.4)$ \\
\hline Female & $154(47.4)$ & $83(77.6)$ \\
\hline \multicolumn{3}{|l|}{ Ethnicity } \\
\hline Caucasian & $157(48.3)$ & $56(52.3)$ \\
\hline Asian & $9(2.8)$ & $4 \quad(3.7)$ \\
\hline Black or African American & $30 \quad(9.2)$ & $27(25.2)$ \\
\hline Unknown & $129(39.7)$ & $20(18.7)$ \\
\hline \multicolumn{3}{|l|}{ Pathologic stage } \\
\hline Stage I & $63(19.4)$ & $14(13.1)$ \\
\hline Stage II & $127(39.1)$ & $38(35.5)$ \\
\hline Stage III & $85(26.2)$ & $31(29.0)$ \\
\hline Stage IV & $39(12.0)$ & $22(20.6)$ \\
\hline Unknown & $11 \quad(3.4)$ & $2(1.9)$ \\
\hline \multicolumn{3}{|l|}{ Pathologic T stage } \\
\hline $\mathrm{T} 1$ & $6 \quad(1.8)$ & $3(2.8)$ \\
\hline $\mathrm{T} 2$ & $66(20.3)$ & $13(12.1)$ \\
\hline $\mathrm{T} 3$ & $214(65.8)$ & $77(72)$ \\
\hline $\mathrm{T} 4$ & $39(12)$ & $14(13.1)$ \\
\hline \multicolumn{3}{|l|}{ Pathologic M stage } \\
\hline M0 & $251(77.2)$ & $67(62.6)$ \\
\hline M1 & $48(14.8)$ & $32(29.9)$ \\
\hline MX & $35(10.8)$ & $18(16.8)$ \\
\hline \multicolumn{3}{|l|}{ Pathologic N stage } \\
\hline N0 & $200(61.5)$ & $57(53.3)$ \\
\hline N1 & 68 (20.9) & $29(27.1)$ \\
\hline N2 & $58(17.8)$ & $21(19.6)$ \\
\hline \multicolumn{3}{|l|}{ Survival status } \\
\hline Alive & 279 (85.8) & $75(70.1)$ \\
\hline Dead & $46(14.2)$ & $32(29.9)$ \\
\hline
\end{tabular}

Values are expressed as n (\%). HIM, high immune score; LIM, low immune score.

LIM samples were not significant $(\mathrm{P}>0.05)$. Fig. 2A presents the immune cell distribution in the HIM samples. Of note, the analysis indicated that gamma delta T cells were not present in any of the samples.

Differences in the proportion of TIICs between the normal tissue samples and HIM samples were then analyzed. Compared with the normal tissues, there were significant changes in the proportion of 15 TIICs in the HIM samples (Fig. 2B). In the HIM samples, naïve B cells, memory B cells, plasma cells, activated natural killer $(\mathrm{NK})$ cells, monocytes, M2 macrophages, resting mast cells and eosinophils decreased significantly, while activated memory $\mathrm{CD}^{+}$, follicular helper
T cells, regulatory T cells, resting NK cells, M0 macrophages, M1 macrophages and activated mast cells were significantly increased.

Prognostic value of immune infiltration in HIM. To determine the prognostic capacity of TIICs in CRC, a survival analysis was performed based on the proportion of immune cells in HIM samples. It was indicated that resting memory $\mathrm{CD}^{+}$ $\mathrm{T}$ cells and regulatory $\mathrm{T}$ cell levels were associated with survival in patients with CRC (Fig. 2C). Of note, patients with $\mathrm{CRC}$ and low proportions of resting memory $\mathrm{CD} 4^{+} \mathrm{T}$ cells had significantly longer survival times than the patients with high proportions.

To further investigate whether TIICs are involved in the development and progression of CRC, the HIM samples were divided into several subgroups based on the pathological TNM stage $\left(\mathrm{T}_{3}+\mathrm{T}_{4}\right.$ vs. $\mathrm{T}_{1}+\mathrm{T}_{2}, \mathrm{~N}_{2}+\mathrm{N}_{3}$ vs. $\mathrm{N}_{0}+\mathrm{N}_{1}, \mathrm{M}_{1}$ vs. $\left.\mathrm{M}_{0}\right)$ and pathological stage (I-II vs. III-IV). Comparative analyses of the TIIC proportions indicated that plasma cells demonstrated a statistically significant association with the pathological $\mathrm{T}$ stage $(\mathrm{P}=0.037)$. Furthermore, M1 macrophages were significantly associated with multiple types of clinical stage (Fig. 2D).

Mutation burden in relation to immune scores. A high mutation burden is one of the characteristics of malignant tumors. To obtain the burden of non-synonymous mutations in HIM and LIM samples, the somatic mutations detected by the mutect 2 software in the two groups were analyzed. The median non-synonymous mutation burden was 111.5 in HIM samples and 96 in LIM samples (Fig. S1). The mutational patterns of the highly mutated genes were distinct between HIM and LIM. As presented in Fig. 3A and B, APC regulator of WNT signaling pathway (APC), tumor protein $\mathrm{p} 53$ (TP53), titin (TTN), KRAS proto-oncogene (KRAS) and phosphatidylinositol-4, 5-bisphosphate 3-kinase catalytic subunit $\alpha$ (PIK3CA) were among the top 10 mutated genes in HIM and LIM samples. It was further identified that most of the mutations were single base substitutions and the substitution of $\mathrm{C}->\mathrm{T}$ was the most common type in all samples (Fig. 3C and D).

Neoantigen burden in association with immune scores. It was also investigated whether the mutational/neoantigen patterns were associated with the immune scores. The neoantigen counts and neoantigen origin protein counts for each CRC sample were obtained from the TCIA database. The LIM samples had a significantly lower neoantigen burden (Wilcoxon rank-sum test $\mathrm{P}=0.0068$; Fig. $3 \mathrm{E}$ ) and neoantigen origin protein burden $(\mathrm{P}=0.0051$; Fig. $3 \mathrm{~F})$ than the samples from the HIM group, which suggested that the HIM samples had a higher number of mutations accumulated in the tumor cell genome than the LIM samples, resulting in a corresponding increase in neoantigen burden and thereby activating more $\mathrm{T}$ cells and producing a stronger immune response.

Comparison of gene expression profiles with immune scores. To reveal the correlation between gene expression profiles and immune scores, a differential analysis of the count data of the genes in the samples from the HIM and LIM groups was performed. As indicated in the volcano plots in Fig. 4A, 

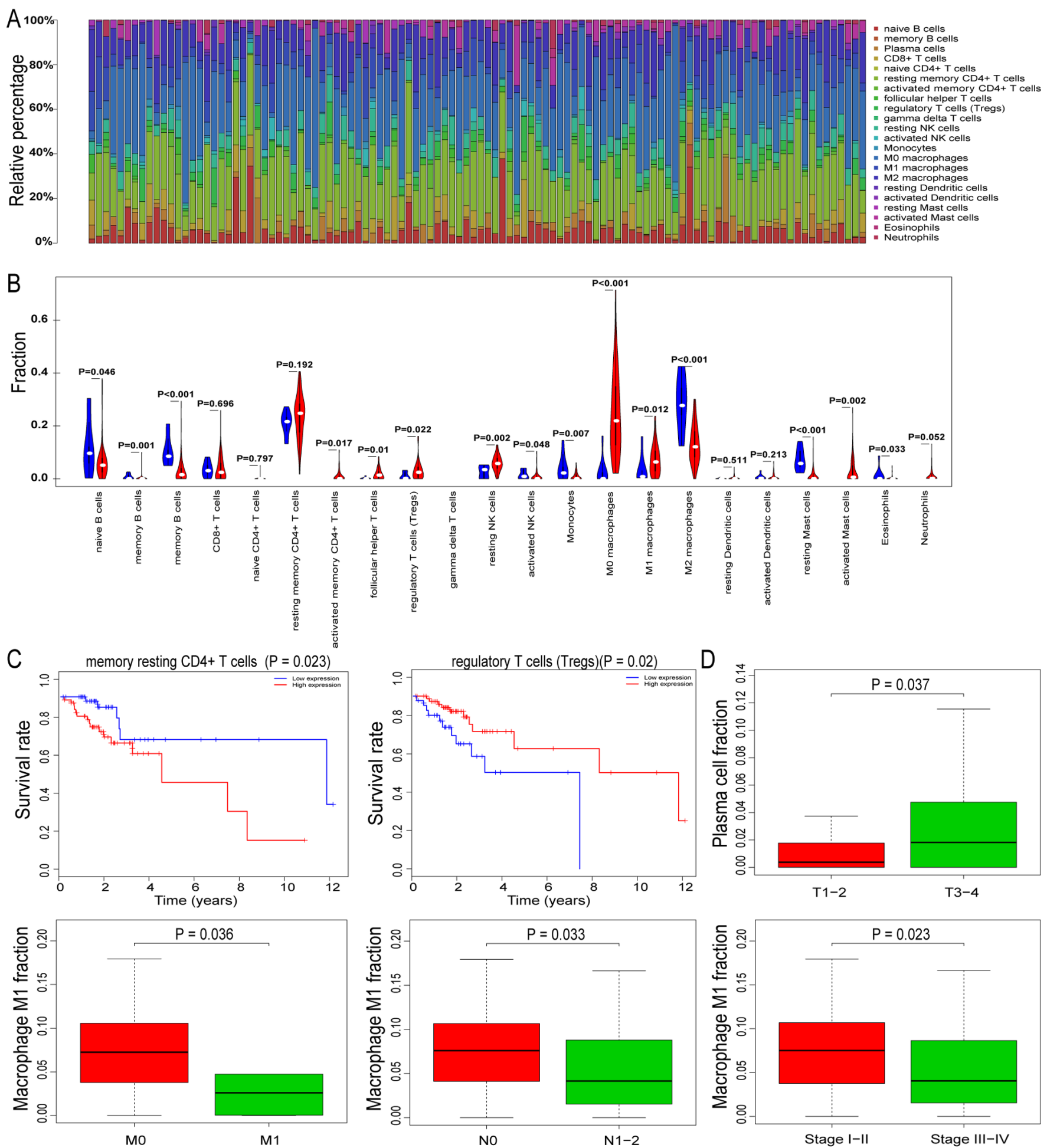

Figure 2. Landscape and prognostic analysis of immune infiltration in HIM. (A) Bar graph summarizing the immune cell subset proportions in the HIM samples. (B) Differences in the proportion of TIICs between the normal tissue samples and HIM samples. (C) Survival plots for certain immune cell types. The depicted P-values are from the log-rank tests. (D) TIICs in association with different stages of progression of colorectal cancer in HIM. NK, natural killer; HIM, high immune score; TIIC, tumor-infiltrating immune cell.

80 genes were upregulated and 1,630 genes were downregulated in the high score group compared with the low score group ( $\log _{2}$ fold changel $>1.5$ and FDR $<0.05$ ). A total of 283 differentially expressed genes were identified from 1,378 immune-associated genes. To outline the potential function of the differentially expressed genes, functional enrichment (GO and KEGG) analysis of the 283 genes was performed (Figs. 4B and S2). The aforementioned genes were enriched in some immune-related pathways, such as B cell receptor signaling pathway, Natural killer cell mediated cytotoxicity and Fc gamma R-mediated phagocytosis. Besides, they were also enriched in disease-related pathways, such as transcriptional misregulation in cancer and inflammatory bowel disease (Figs. 4B and S2). 

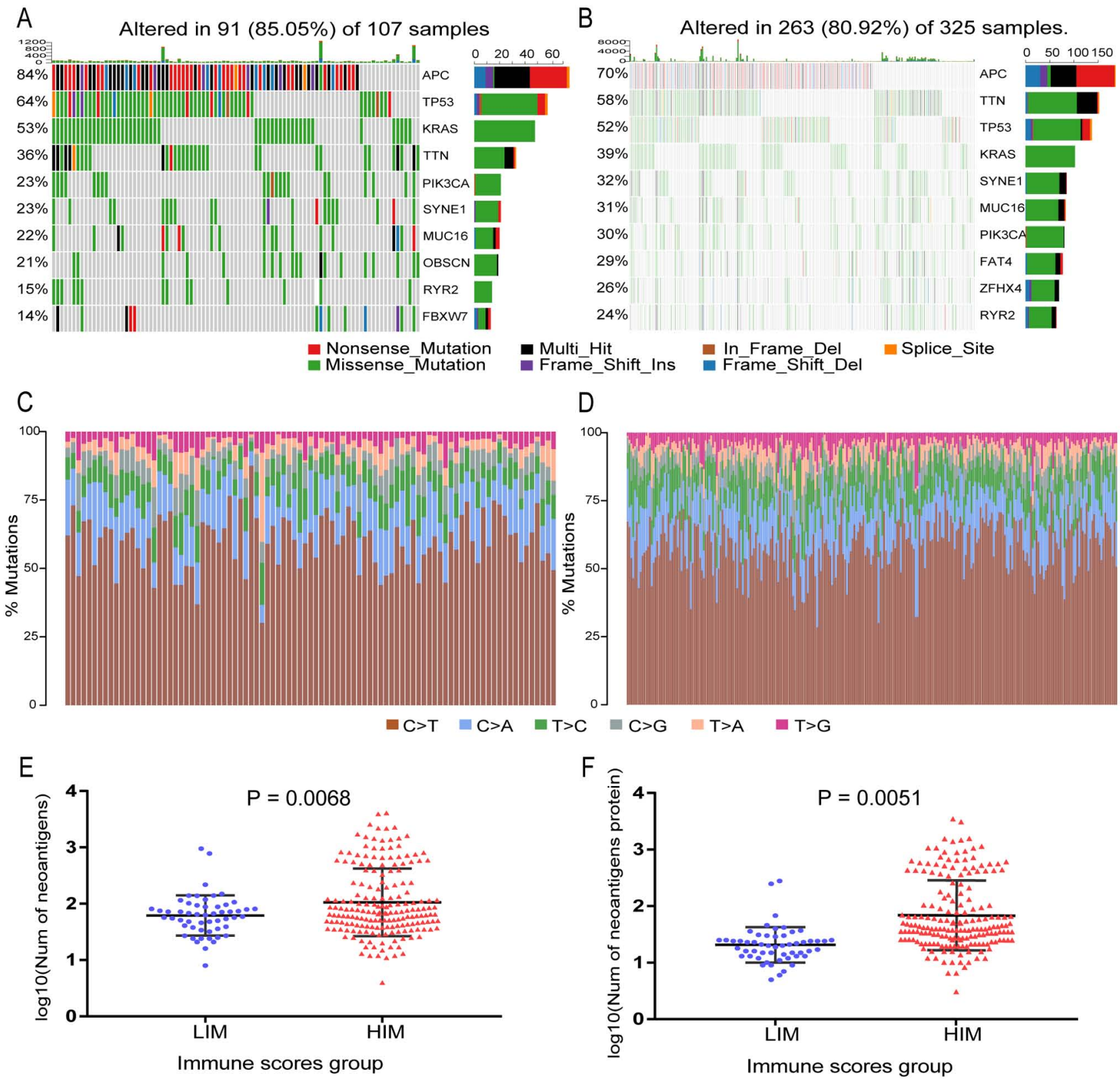

Figure 3. Landscape of mutations in colorectal cancer and a comparison of neoantigens between HIM and LIM samples, including the top 10 most mutated genes for (A) LIM samples and (B) HIM samples. The right stacked bar graph of each figure displays the number of variant types. Substitution distribution for each (C) LIM sample and (D) HIM sample. (E) Plots of the number of neoantigen peptides. (F) Plots of the number of Neoantigen-related proteins. Graph indicating a significant difference in neoantigen burden between HIM and LIM samples $(\mathrm{P}<0.05)$. HIM, high immune score; LIM, low immune score; Ins, insertion; Del, deletion; Num, number; APC, APC regulator of WNT signaling pathway; TP53, tumor protein p53; KRAS, KRAS proto-oncogene, GTPase; TTN, titin; PIK3CA, phosphatidylinositol-4, 5-bisphosphate 3-kinase catalytic subunit alpha; SYNE1, spectrin repeat containing nuclear envelope protein 1; MUC16, mucin 16, cell surface associated; OBSCN, obscurin, cytoskeletal calmodulin and titin-interacting RhoGEF; RYR2, ryanodine receptor 2; FBXW7, F-box and WD repeat domain containing 7; FAT4, FAT atypical cadherin 4; ZFHX4, zinc finger homeobox 4.

Potential function of differentially expressed genes. Kaplan-Meier curve analysis with the log-rank test for each of the 283 genes provided 13 genes whose expression was significantly correlated with the survival of the patients with CRC. To investigate the functional association of the 13 genes, the genes were analyzed using the GeneMANIA plugin of Cytoscape to generate an interaction network (Fig. 4C). Most of the network interactions were coexpression. The Kaplan-Meier survival curves that were significantly different for the 13 genes (high vs. low expression) are presented in Figs. 4D and S3. Further clinical analysis of these genes demonstrated that the SERPINE1 and UCHL1 were significantly associated with multiple types of clinical stage (Fig. 4E). The association between the expression profiles of these two genes and TIICs was also analyzed and it was revealed that their copy number variation was significantly associated with changes in the proportion of multiple types of TIIC, such as B cells, CD8 ${ }^{+}$ T cells, neutrophils and dendritic cells (Fig. S4).

Comprehensive validation in GEO datasets. To examine the universality of the results from the TCGA cohort, the GSE17536 and GSE21510 datasets were analyzed for validation. After 


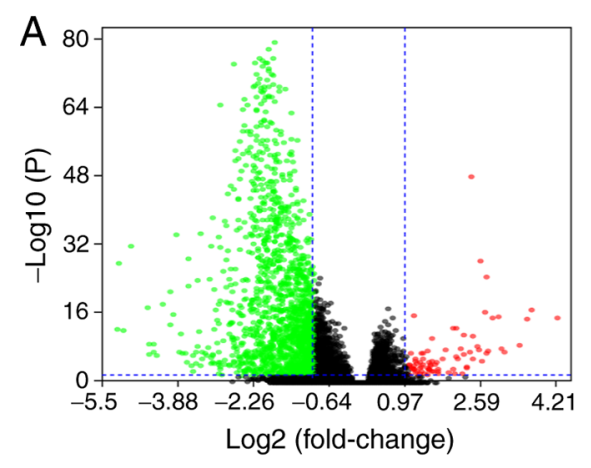

B

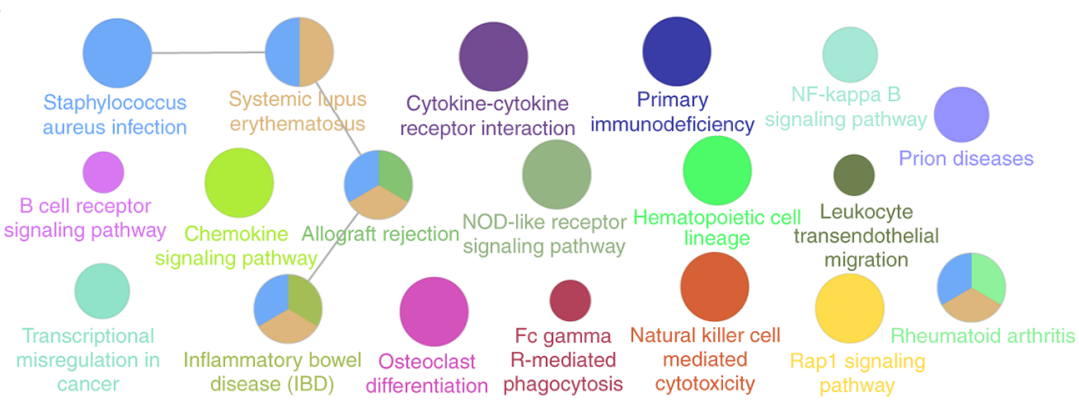

D
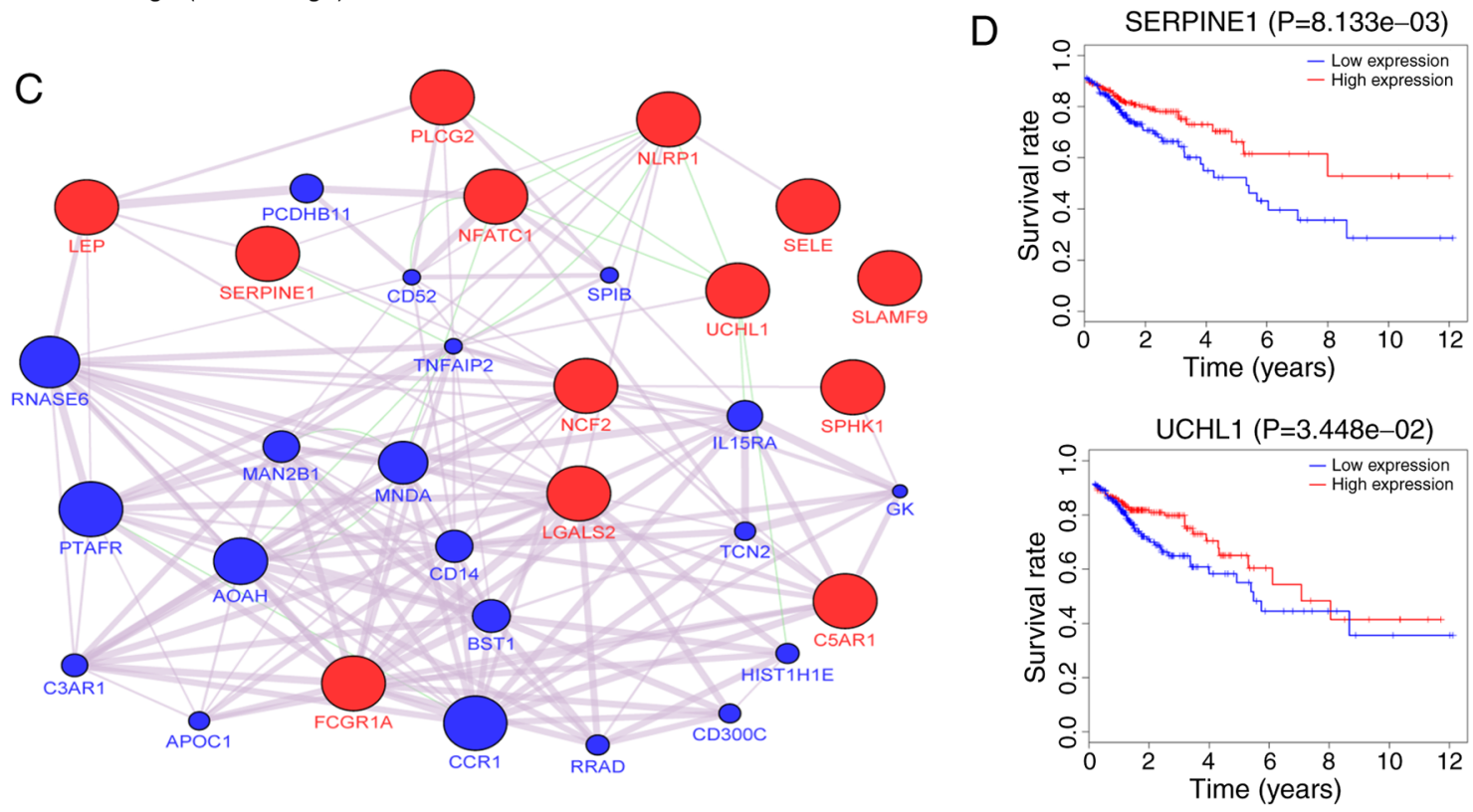

E
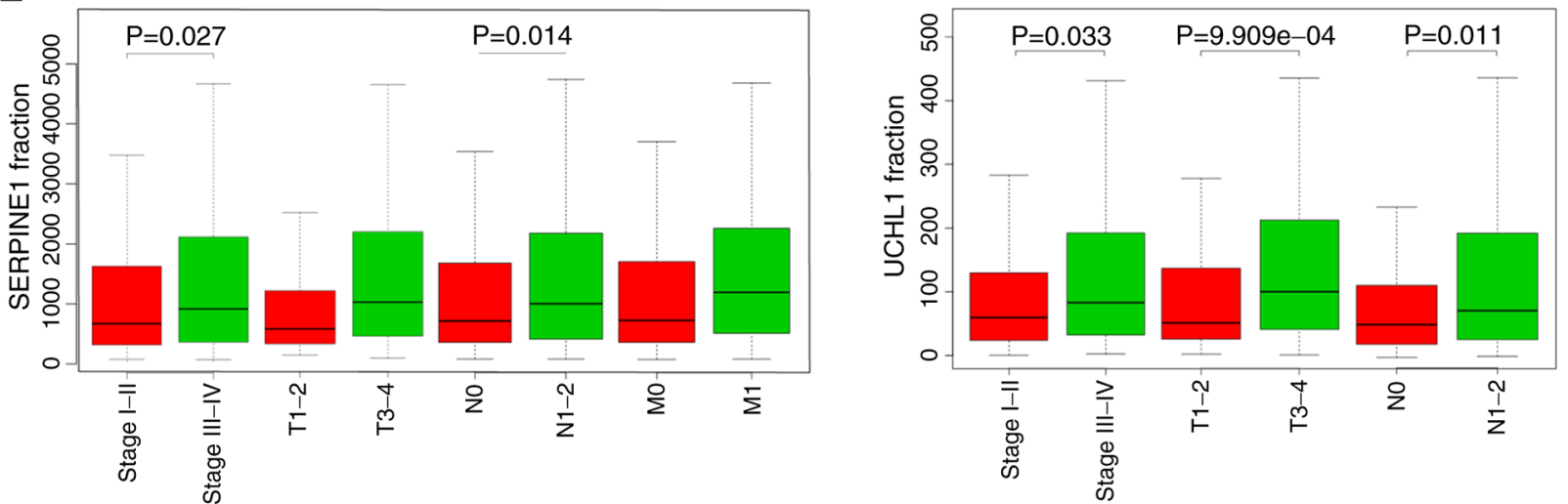

Figure 4. Gene expression spectrum. (A) Volcano plot of differentially expressed genes between HIM and LIM samples. The three colored dots represent different types of mRNAs, among which green represents a significant downregulation, red represents a significant upregulation and black represents no significant differential expression. (B) Kyoto Encyclopedia of Genes and Genomes pathway analysis of 283 differentially expressed genes. (C) GeneMANIA network of 13 genes significantly correlated with the survival of patients with colorectal cancer. The query genes are red and their interacting genes are blue. (D) Survival analysis for SERPINE1 and UCHL1. The red lines denote high expression of the gene and the blue lines denote low expression. (E) SERPINE1 and UCHL1 are related to the progression of CRC. SERPINE1, serpin family Emember 1; UCHL1, ubiquitin C-terminal hydrolase L1; LEP, leptin; PLCG2, phospholipase C gamma 2; NLRP1, NLR family pyrin domain containing 1; SELE, selectin E; SERPINE1, serpin family E member 1; NFATC1, nuclear factor of activated T cells 1; SLAMF9, SLAM family member 9; UCHL1, ubiquitin C-terminal hydrolase L1; NCF2, neutrophil cytosolic factor 2; SPHK1, sphingosine kinase 1; LGALS2, galectin 2; C5AR1, complement C5a receptor 1; FCGR1A, Fc fragment of IgG receptor Ia; PCDHB11, protocadherin beta 11; CD52, CD52 molecule; SPIB, Spi-B transcription factor; RNASE6, ribonuclease A family member k6; TNFAIP2, TNF alpha induced protein 2; PTAFR, platelet activating factor receptor; MNDA, myeloid cell nuclear differentiation antigen; IL15RA, nterleukin 15 receptor subunit alpha; AOAH, acyloxyacyl hydrolase; CD14, CD14 molecule; BST1, bone marrow stromal cell antigen 1; C3AR1, complement C3a receptor 1; CCR1, C-C motif chemokine receptor 1; CD300C, CD300c molecule; GK, glycerol kinase.

batch correction (Fig. S5A), the same cutoff as that for the TCGA sample analysis was used to stratify all GEO samples into HIM and LIM groups. Due to the particularity of HIM samples, the CIBERSORT algorithm was used to assess the difference in the proportion of 22 immune cell subsets in these samples (Fig. S5B). It was revealed that the proportions of the immune cell subsets in the TCGA and GEO datasets were similar. In addition, differential analysis of gene count data in 
samples with HIM and LIM revealed that SERPINE1 was still significantly differentially expressed ( $\log _{2}$ fold changel $>1.5$, $\mathrm{P}=0.00142$ ). However, the difference in UCHL1 expression was not significant.

\section{Discussion}

The present study sought to identify tumor microenvironment-associated factors that contribute to the OS of patients with CRC with HIM or LIM. The results indicated that MSIH tumors rather than MSS tumors were significantly associated with high immune scores. A previous study indicated that in CRC, MSIH tumors have 10s of times more somatic mutations than MSS tumors (26). Under these conditions, lymphocyte infiltration is prominent due to increased neoantigen burdens and more stroma cells are present in tumor tissues (27). In addition, the cytolytic activity of tumor cells is positively correlated with the neoantigen burden (15). In line with this, the present results suggested that the HIM samples were characterized by higher cytolytic activity and stromal scores than LIM samples. This means that the samples in the HIM group had a good cytolytic immune response and relatively abundant stromal cells due to the higher levels of TIICs. In addition, a previous study indicated that cytolytic activity was associated with immune responses and improved prognoses (28). The present study also indicated significantly improved OS in patients with HIM vs. LIM.

Using CIBERSORT, the proportional changes of the 22 immune cell subsets in the HIM and LIM samples were analyzed. The TIIC proportions of the samples in LIM did not meet the criterion of $\mathrm{P}<0.05$, which was due to their comparatively lower immune score. It is worth noting that the P-value obtained with CIBERSORT only reflects one part of a sample that contains immune cells and non-immune cells. Therefore, $\mathrm{P}>0.05$ does not mean that TIICs do not exist in LIM samples. However, the present results indicated that the proportion of TIICs was not significant in samples with lower immune scores. Tumor-associated microenvironments, which include immune cells, are able to inhibit malignant cells. Numerous studies have indicated that the degree of infiltration of immune cells, tissue localization and cell type are significantly associated with CRC progression and survival. For instance, the 5-year OS values for stage III CRC patients with lower levels of TIICs were determined to be significantly lower than those for stage III CRC patients with high levels of TIICs (29).The present study also indicated that HIM samples have significant changes in the proportion of TIIC and the survival time of patients with CRC with high immune scores was significantly improved. In addition, the present results provided details on the infiltration of the 22 TIIC subsets in CRC. The proportions of resting memory $\mathrm{CD}^{+} \mathrm{T}$ cells and macrophages were the highest, while gamma delta $\mathrm{T}$ cells were not present in any of the samples. The present study also indicated that resting $\mathrm{CD} 4^{+}$ memory $\mathrm{T}$ cells were significantly associated with the survival of patients with CRC. Resting $\mathrm{CD}^{+}$memory $\mathrm{T}$ cells may help $\mathrm{CD}^{+} \mathrm{T}$ cells inhibit tumors and block $\mathrm{CD}^{+} \mathrm{T}$-cell activation and NK cell activity (30). The present study also confirmed that they have a key role in the development of CRC.

The neoantigen burden is an effective biomarker in cancer immunotherapy and neoantigens may be the focus of the development of novel therapeutic approaches to modify T-cell reactivity against this class of antigens (31). The probability of the presence of $\mathrm{CD}^{+} \mathrm{T}$ cells in cancer lesions is higher in tumors with high mutation burdens than in those with low mutation burdens (32). T-cell reactivity against neoantigens is common in melanoma (33). In CRC, the present study indicated that the neoantigen peptide burden of HIM samples was significantly higher than that of LIM samples. More than one neoantigen peptide may be derived from a protein. In the HIM sample, the proportions of infiltration of activated memory $\mathrm{CD} 4^{+} \mathrm{T}$ cells were significantly increased. One reason is that the HIM samples have a high neoantigen burden and studies have also indicated that most of the new antigen-specific T-cell responses in melanoma are against neoantigens (34).

The present study indicated that certain genes were highly mutated in both HIM and LIM samples. The tumor suppressor gene TP53 exhibited a discontinuous mutation distribution in the two groups. TP53 has a regulatory role in cell proliferation and apoptosis (35). The loss of TP53 may lead to CRC development and progression through a multistep process (36). An association between TP53 mutations and worse outcome in stage III CRC has been reported (37). The present study indicated that 68 (64\%) LIM samples had TP53 gene mutations. The number of correspondingly mutated HIM samples was 169 (52\%). Of note, the APC gene had the highest mutation frequency in HIM and LIM samples, at 84 and $70 \%$, respectively. In addition, the CRC proto-oncogene KRAS was mutated in 39 and 53\% of HIM and LIM samples, respectively, and its mutations cause the RAS/RAF/MAPK pathways to remain active with loss of normal regulation of cell growth (38).

By comparing the gene expression profiles in HIM and LIM samples and screening immune-associated genes, 283 tumor microenvironment-related genes were identified in the present study. Functional enrichment analyses indicated that these genes mainly participated in the immune response and cell adhesion. The study further focused on SERPINE1 and UCHL1, which are immune-associated genes that were significantly differentially expressed between HIM and LIM samples, and their expression was significantly associated with OS and multiple types of clinical stage in patients with CRC. In addition, the present analysis revealed that their copy number variation led to significant changes in infiltration of the multiple immune cell subsets. Previous studies have indicated that SERPINE1 overexpression occurs in primary tumors caused by KRAS mutations in CRC (39) and UCHL1 is associated with lymph node metastasis in CRC (40). Therefore, it was concluded that SERPINE1 and UCHL1 may be additional biomarkers for CRC. The difference in expression of SERPINE1 was also verified in the GEO dataset, however, the difference in UCHL1 expression was not significant. How they affect tumor progression in tumor microenvironments requires further investigation. However, the present study had certain limitations, as all of the results were obtained in silico. Further in vivo or clinical studies will contribute to the further elucidation of the relationship between immune scores and microenvironmental changes in CRC.

In conclusion, in CRC samples that had high immune scores, a good cytolytic immune response and relatively abundant stromal cells, significant infiltration of 22 immune 
cell subsets and a high non-synonymous mutation burden and neoantigen burden was identified, and a set of genes associated with the tumor microenvironment was extracted. The present study revealed that a tumor microenvironment of CRC with a high immune score is associated with favorable survival.

\section{Acknowledgements}

Not applicable.

\section{Funding}

This work was partly supported by the Shanghai Natural Science Foundation (grant no. 15ZR1420800 to SP) and the Quality Engineering Project of Colleges and Universities in Anhui Province (grant nos. 2015zytz070 and 2019rcsfjd088 to $\mathrm{XH}$ ).

\section{Availability of data and materials}

The datasets used and/or analyzed during the current study are available from the corresponding author on reasonable request.

\section{Authors' contributions}

WY and SP conceived the study, collected the data and performed the bioinformatics analyses. WC and $\mathrm{XH}$ performed quality control of the raw data and participated in part of the data analyses. WY and SP wrote the manuscript and supervised the study. All authors read and approved the final manuscript.

\section{Ethics approval and consent to participate}

Not applicable.

\section{Patient consent for publication}

Not applicable.

\section{Competing interests}

The authors declare that they have no competing interests.

\section{References}

1. Bray F, Ferlay J, Soerjomataram I, Siegel RL, Torre LA and Jemal A: Global cancer statistics 2018: GLOBOCAN estimates of incidence and mortality worldwide for 36 cancers in 185 countries. CA Cancer J Clin 68: 394-424, 2018.

2. Marks KM, West NP, Morris E and Quirke P: Clinicopathological, genomic and immunological factors in colorectal cancer prognosis. Br J Surg 105: e99-e109, 2018.

3. Nancy K: The management of resectable and unresectable liver metastases from colorectal cancer. Curr Opin Oncol 22: 364-373, 2010.

4. Lupinacci RM, Andraus W, De Paiva Haddad LB, Carneiro D Albuquerque LA and Herman P: Simultaneous laparoscopic resection of primary colorectal cancer and associated liver metastases: A systematic review. Tech Coloproctol 18: 129-135, 2014.

5. Dougan M and Dranoff G: Immune therapy for cancer. Annu Rev Immunol 27: 83-117, 2009.

6. Hanahan D and Coussens LM: Accessories to the crime: Functions of cells recruited to the tumor microenvironment. Cancer Cell 21: 309-322, 2012.
7. Charoentong P, Finotello F, Angelova M, Mayer C, Efremova M, Rieder D, Hackl H and Trajanoski Z: Pan-cancer immunogenomic analyses reveal genotype-immunophenotype relationships and predictors of response to checkpoint blockade. Cell Rep 18: 248-262, 2017.

8. Alexandrov LB, Nik-Zainal S, Wedge DC, Aparicio SA, Behjati S, Biankin AV, Bignell GR, Bolli N, Borg A, Børresen-Dale AL, et al: Signatures of mutational processes in human cancer. Nature 500: 415-421, 2013.

9. Le DT, Durham JN, Smith KN, Wang H, Bartlett BR, Aulakh LK, Lu S, Kemberling H, Wilt C, Luber BS, et al: Mismatch repair deficiency predicts response of solid tumors to PD-1 blockade. Science 357: 409-413, 2017.

10. Pastille E, Wasmer MH, Adamczyk A, Vu VP, Mager LF, Phuong NNT, Palmieri V, Simillion C, Hansen W, Kasper S, et al: The IL-33/ST2 pathway shapes the regulatory T cell phenotype to promote intestinal cancer. Mucosal Immunol 12: 990-1003, 2019.

11. Yoshihara K, Shahmoradgoli M, Martínez E, Vegesna R, Kim H, Torres-Garcia W, Treviño V, Shen H, Laird PW, Levine DA, et al: Inferring tumour purity and stromal and immune cell admixture from expression data. Nat Commun 4: 2612, 2013.

12. Priedigkeit N, Watters RJ, Lucas PC, Basudan A, Bhargava R, Horne W, Kolls JK, Fang Z, Rosenzweig MQ, Brufsky AM, et al: Exome-capture RNA sequencing of decade-old breast cancers and matched decalcified bone metastases. JCI Insight 2: e95703, 2017.

13. Jia D, Li S, Li D, Xue H, Yang D and Liu Y: Mining TCGA database for genes of prognostic value in glioblastoma microenvironment. Aging (Albany NY) 10: 592-605, 2018.

14. Shah N, Wang P, Wongvipat J, Karthaus WR, Abida W, Armenia J, Rockowitz S, Drier Y, Bernstein BE, Long HW, et al: Regulation of the glucocorticoid receptor via a BET-dependent enhancer drives antiandrogen resistance in prostate cancer. Elife 6: e27861, 2017.

15. Rooney MS, Shukla SA, Wu CJ, Getz G and Hacohen N: Molecular and genetic properties of tumors associated with local immune cytolytic activity. Cell 160: 48-61, 2015.

16. Hause RJ, Pritchard CC, Shendure J and Salipante SJ: Corrigendum: Classification and characterization of microsatellite instability across 18 cancer types. Nat Med 23: 1241, 2017.

17. Eide PW, Bruun J, Lothe RA and Sveen A: CMScaller: An R package for consensus molecular subtyping of colorectal cancer pre-clinical models. Sci Rep 7: 16618, 2017.

18. Bhattacharya S, Andorf S, Gomes L, Dunn P, Schaefer H, Pontius J, Berger P, Desborough V, Smith T, Campbell J, et al: ImmPort: Disseminating data to the public for the future of immunology. Immunol Res 58: 234-239, 2014.

19. Mayakonda A and Koeffler HP: Maftools: Maftools: Efficient analysis, visualization and summarization of MAF files from large-scale cohort based cancer studies. BioRxiv: 052662, 2016.

20. Newman AM, Liu CL, Green MR, Gentles AJ, Feng W, Xu Y, Hoang CD, Diehn M and Alizadeh AA: Robust enumeration of cell subsets from tissue expression profiles. Nat Methods 12: 453-457, 2015.

21. Robinson MD, McCarthy DJ and Smyth GK: edgeR: A bioconductor package for differential expression analysis of digital gene expression data. Bioinformatics 26: 139-140, 2010.

22. Li T, Fan J, Wang B, Traugh N, Chen Q, Liu JS, Li B and Liu XS: TIMER: A web server for comprehensive analysis of tumor-infiltrating immune cells. Cancer Res 77: e108: e110, 2017.

23. Smoot ME, Ono K, Ruscheinski J, Wang PL and Ideker T: Cytoscape 2.8: New features for data integration and network visualization. Bioinformatics 27: 431-432, 2011.

24. Montojo J, Zuberi K, Rodriguez H, Kazi F, Wright G, Donaldson SL, Morris Q and Bader GD: GeneMANIA Cytoscape plugin: Fast gene function predictions on the desktop. Bioinformatics 26: 2927-2928, 2010.

25. Lin $\mathrm{H}$ and Zelterman D: Modeling survival data: Extending the Cox model. Technometrics 44: 85-86, 2002.

26. Park JH, Powell AG, Roxburgh CS, Horgan PG, Mcmillan DC and Edwards J: Mismatch repair status in patients with primary operable colorectal cancer: Associations with the local and systemic tumour environment. Br J Cancer 114: 562-570, 2016.

27. Giannakis M, Mu XJ, Shukla SA, Qian ZR, Cohen O, Nishihara R, Bahl S, Cao Y, Amin-Mansour A, Yamauchi M, et al: Genomic correlates of immune-cell infiltrates in colorectal carcinoma. Cell Rep 15: 857-865, 2016. 
28. Narayanan S, Kawaguchi T, Yan L, Peng X, Qi Q and Takabe K: Cytolytic activity score to assess anticancer immunity in colorectal cancer. Ann Surg Oncol 25: 2323-2331, 2018.

29. Huh JW, Lee JH and Kim HR: Prognostic significance of tumor-infiltrating lymphocytes for patients with colorectal cancer. Arch Surg 147: 366-372, 2012

30. Rosenberg $\mathrm{J}$ and Huang J: $\mathrm{CD}^{+} \mathrm{T}$ cells and NK cells: Parallel and complementary soldiers of immunotherapy. Curr Opin Chem Eng 19: 9-20, 2018.

31. Schumacher TN and Schreiber RD: Neoantigens in cancer immunotherapy. Science 348: 69-74, 2015.

32. Brown SD, Warren RL, Gibb EA, Martin SD, Spinelli JJ, Nelson BH and Holt RA: Neo-antigens predicted by tumor genome meta-analysis correlate with increased patient survival. Genome Res 24: 743-750, 2014.

33. Kvistborg P, Shu CJ, Heemskerk B, Fankhauser M, Thrue CA, Toebes M, van Rooij N, Linnemann C, van Buuren MM, Urbanus JH, et al: TIL therapy broadens the tumor-reactive CD8(+) $\mathrm{T}$ cell compartment in melanoma patients. Oncoimmunology 1: $409-418,2012$.

34. Linnemann C, van Buuren MM, Bies L, Verdegaal EM, Schotte R, Calis JJ, Behjati S, Velds A, Hilkmann H, Atmioui DE, et al: High-throughput epitope discovery reveals frequent recognition of neo-antigens by $\mathrm{CD}^{+} \mathrm{T}$ cells in human melanoma. Nat Med 21: 81-85, 2015.

35. Perego P, Giarola M, Righetti SC, Supino R, Caserini C, Delia D, Pierotti MA, Miyashita T, Reed JC and Zunino F: Association between cisplatin resistance and mutation of p53 gene and reduced bax expression in ovarian carcinoma cell systems. Cancer Res 56: 556-562, 1996.
36. Fearon ER and Vogelstein B: A genetic model for colorectal tumorigenesis. Cell 61: 759-767, 1990.

37. Westra JL, Michael S, Harry H, de Boer JP, Kraak MM, de Jong D, ter Elst A, Mulder NH, Buys CH, Hofstra RM and nPlukker JT: Determination of TP53 mutation is more relevant than microsatellite instability status for the prediction of disease-free survival in adjuvant-treated stage III colon cancer patients. J Clin Oncol 23: 5635-5643, 2005.

38. Borrero-Palacios A, Cebrián A, Gómez Del Pulgar MT, García-Carbonero R, Garcia-Alfonso P, Aranda E, Elez E, López-López R, Cervantes A, Valladares M, et al: Combination of KIR2DS4 and Fc $\gamma$ RIIa polymorphisms predicts the response to cetuximab in KRAS mutant metastatic colorectal cancer. Sci Rep 9: 2589, 2019.

39. Alamo P, Gallardo A, Di Nicolantonio F, Pavón MA, Casanova I, Trias M, Mangues MA, Lopez-Pousa A, Villaverde A, Vázquez E, et al: Higher metastatic efficiency of KRas G12V than KRas G13D in a colorectal cancer model. FASEB J 29: 464-476, 2015.

40. Zhong J, Zhao M, Ma Y, Luo Q, Liu J, Wang J, Yuan X, Sang J and Huang C: UCHL1 acts as a colorectal cancer oncogene via activation of the $\beta$-catenin/TCF pathway through its deubiquitinating activity. Int J Mol Med 30: 430-436, 2012.

This work is licensed under a Creative Commons Attribution-NonCommercial-NoDerivatives 4.0 International (CC BY-NC-ND 4.0) License. 